\title{
MEMS tunable capacitors with fragmented electrodes and rotational electro-thermal drive
}

\author{
A. Mehdaoui $\cdot$ M. B. Pisani $\cdot$ D. Tsamados $\cdot$ \\ F. Casset $\cdot$ P. Ancey $\cdot$ A. M. Ionescu
}

Received: 29 June 2006/ Accepted: 20 November 2006/Published online: 5 January 2007

(C) Springer-Verlag 2007

\begin{abstract}
This paper reports on the design, simulation and fabrication of tunable MEMS capacitors with fragmented metal (AlSi $4 \%$ ) electrodes. We examine a rotational electro-thermal actuation. An analytic model of the rotational effect thermal actuator was established in order to show the periodicity of the capacitance when the angle increases. Evaluation of the impact of fringing fields on the capacitance has been carried out using finite element analysis (FEA). The MEMS capacitors were fabricated using metal surface micromachining with polyimide sacrificial layer. The maximum rotation, corresponding to a maximum angle of $7^{\circ}$, was obtained near $1.2 \mathrm{~V}$ and $299 \mathrm{~mA}$. The proposed capacitor has a practical tuning range of $30 \%$. FEA has shown that this figure can be improved with design optimization. The MEMS architecture based on rotational effect and fragmented electrodes does not suffer from the pull in effect and offers a practical solution for future above-IC capacitors.
\end{abstract}

A. Mehdaoui · F. Casset · P. Ancey

ST Microelectronics, Crolles, France
A. Mehdaoui $(\varangle) \cdot$ M. B. Pisani · D. Tsamados
A. M. Ionescu
Electronics Laboratory,
Swiss Federal Institute of Technology,
ELB 311 Building, EPF Lausanne, Switzerland
e-mail: alexandre.mehdaoui@epfl.ch
F. Casset
CEA/LETI, Grenoble, France

\author{
Abbreviations \\ CMP Chemical mechanical planarization \\ FEA Finite element analysis \\ IC Integrated circuit \\ MEMS Micro electro mechanical systems \\ SEM Scanning electron microscope
}

\section{Introduction}

Many types of MEMS tunable capacitors have been proposed over the last years, essentially focusing on new architecture solutions to increase their tuning range. Two or three parallel plate capacitors with vertical electrostatic actuation (air-gap variation) show a limited tuning range due to the pull in effect (non equilibrium between electrostatic and elastic forces, Young and Boser 1996; Dec and Suyama 1998). Some other works (Yoon and Nguyen 2000; Liu and Huang 2001) have demonstrated effective solutions by varying the effective area or dielectric constant of capacitors. This paper reports on the design, simulation fabrication of tunable capacitors with fragmented metal (AlSi $4 \%$ ) electrodes that are rotationally driven by electrothermal actuators and avoid the pull in effect.

The proposed new capacitor is shown in Fig. 1. It is made of two fragmented electrodes: (1) a bottom fixed electrode and (2) a top rotationally movable electrode suspended over a $1.4 \mu \mathrm{m}$ air gap. Both metal electrodes have a circular comb form and the top electrode is rotationally driven by electro-thermal actuators. We used two arms connected on each part of the small central disk structure to rotate the top electrode and 


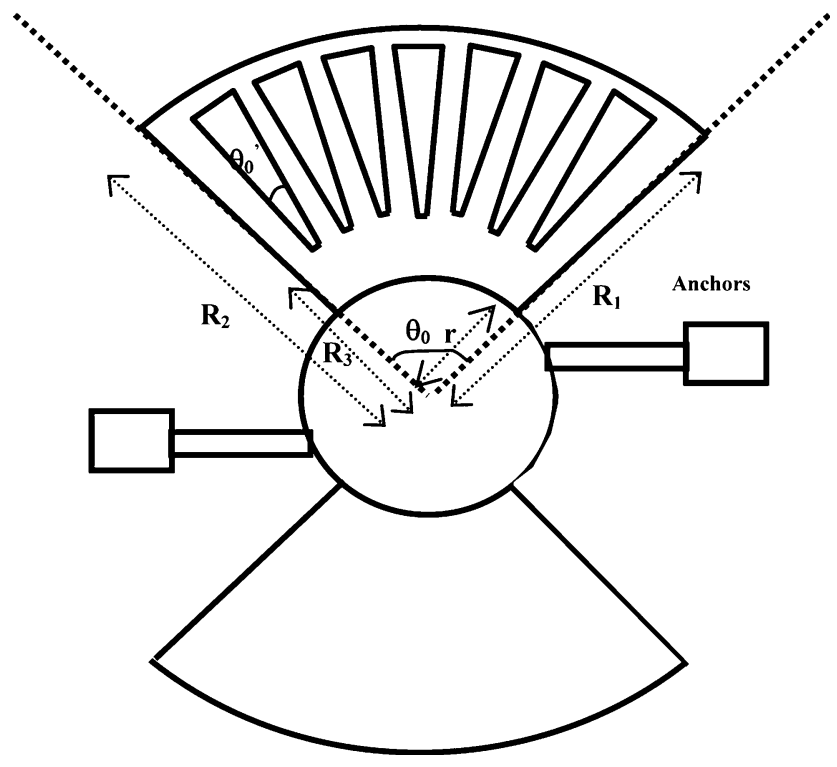

Fig. 1 Rotational effect thermal actuator

change the capacitance. The electro-thermal drive gives more flexibility in terms of design, dimensions and applied voltages. However, this comes at the price of an increased power consumption compared to the classical tunable capacitor configuration.

\section{MEMS capacitor with fragmented electrodes and rotational electro-thermal actuation}

\subsection{Analytic modeling and simulation}

The schematic of a MEMS capacitor with fragmented electrodes using arms electro-thermal actuation is presented in Fig. 2. We used two arms connected on each part of the small central disk structure to rotate the top electrode and change the capacitance. When a

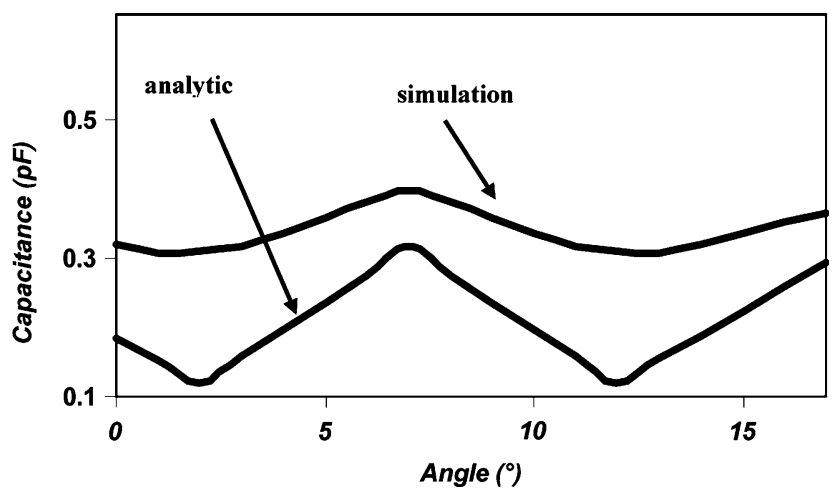

Fig. 2 Capacitance versus angular displacement current passes through the arms, the injected power induces a net thermal expansion of the arm (Eq. 1):

$\Delta l=\alpha l \Delta T$

where $\alpha$ is the thermal expansion, $l$ the length of the beam and $\Delta T$ the thermal gradient that creates a moment that rotates the entire structure. The amplification (effective displacement of the outer disk) depends on the dimensions of the actuator and the ratio between the radius of the inner and outer disk. We have designed electro-thermal actuators with various dimensions and a case study is presented on the following geometry of arms: width $=4 \mu \mathrm{m}$, length $=200 \mu \mathrm{m} \quad$ and $\quad$ thickness $=4 \mu \mathrm{m}$. Figure 1 shows the shape and the area of ach part (fragmented and non fragmented). The effective area of the tunable capacitors has a straightforward dependence on the rotation angle, which can be expressed mathematically, as follows:

$S_{1}=\pi r^{2}$

$S_{2}=\frac{2 \pi\left(R_{2}^{2}-R_{1}^{2}\right)\left(\theta_{0}-\theta_{e}\right)}{360}$

$S_{3}=\frac{2 \pi\left(R_{3}^{2}-r^{2}\right)\left(\theta_{0}-\theta_{e}\right)}{360}$

$S_{4}=\frac{2 \pi\left(R_{2}^{2}-R_{1}^{2}\right)\left(\theta_{0}^{\prime}-\theta_{e}\right) N}{360}$

and the total area is calculated as (by combining Eqs. 2-5):

$S=S_{\mathrm{f}}+S_{\mathrm{nf}}$

$S_{\mathrm{f}}=S_{4}$

$S_{\mathrm{nf}}=S_{1}+S_{2}+S_{3}$,

respectively the area of fragmented and non fragmented surface. The capacitance is expressed in Eq. 9 (by combining Eqs. 6-8):

$C=C_{\mathrm{f}}+C_{\mathrm{nf}}=\frac{\varepsilon_{0}}{d}\left(S_{\mathrm{f}}+S_{\mathrm{nf}}\right)$

$C_{\mathrm{f}}$ and $C_{\mathrm{nf}}$ have, respectively, a periodic and linear variation when the rotational angle increases. $R_{1}=190 \mu \mathrm{m}, R_{2}=210 \mu \mathrm{m}, R_{3}=60 \mu \mathrm{m}, r=10 \mu \mathrm{m}$, $\theta_{0}=85^{\circ}, \theta_{0}{ }^{\prime}=5^{\circ}, d=1.2 \mu \mathrm{m} . \theta_{0}, \theta_{0}{ }^{\prime}$ and $\theta_{e}$ are the initial-state angle of the top electrode, the initial angle of the finger and the rotational angle, respectively. $N$ is the number of fingers. 
The capacitance as a function of $\theta_{e}$ has been studied by finite element analysis (ANSYS ${ }^{\mathrm{TM}}$ ). Figure 2 shows the periodicity of the capacitance when the angle increases, with the minimum value periodically decreasing because of the decrease of $S_{2}$ and $S_{3}$, which are the non fragmented parts of the disk area. We compare the analytical and simulated tuning range results.

For an angular displacement of $7^{\circ}$, we obtained, for the analytical and simulated results, a tuning range more than 70 and $30 \%$, respectively. This discrepancy between analytical and simulated results is due to the fringing capacitance, which is not accounted for in our model.

Moreover this tuning range is limited by the design of the capacitor (area) and the buckling in the arm. By a proper design (minimization of $S 1, S 2$ and $S 3$ and more favorable fragmentation) this can be increased in excess of $100 \%$.

\subsection{Optimization}

The design has been optimized by FEA, using AN$\mathrm{SYS}^{\mathrm{TM}}$ simulator. The optimization goal was to obtain the maximum tuning range. So, the fringing capacitance should be minimized. First, we studied the influence of, $\theta_{0}{ }^{\prime}$, then, the angle between two fingers (pitch), and finally the surface of the non fragmented areas.

\subsubsection{Influence of the angle $\theta_{0}$}

The design presented in Fig. 1 was used as a base for the optimization. First we have tried to increase the tuning range by changing the angle $\theta_{0}{ }^{\prime}$. Each finger is distant by $\theta_{0}^{\prime}$. Figure 3 shows the capacitance versus the angular displacement for different values of $\theta_{0}{ }^{\prime}$.

Table 1 shows the tuning range for these four angles $\theta_{0}{ }^{\prime}$. We can see that the tuning range is optimal for $\theta_{0}^{\prime}=13^{\circ}$ in case of a rotation of $\theta_{e}=13^{\circ}$.

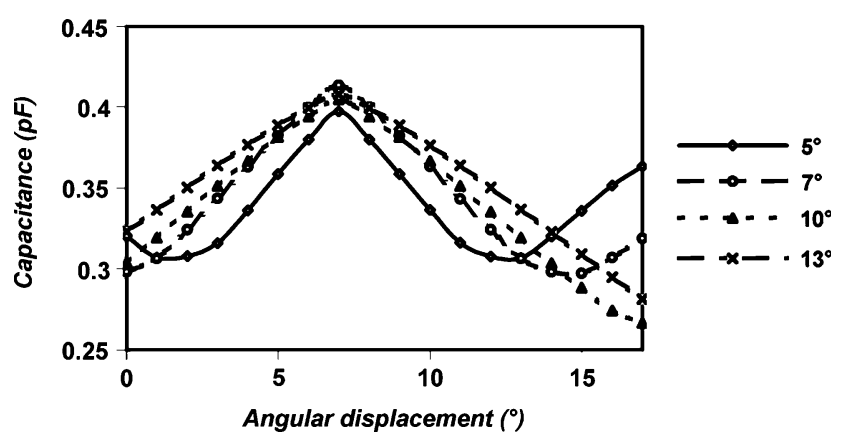

Fig. 3 Capacitance versus angular displacement for different angle asdasdasa $\theta_{0}$
Table 1 Tuning range of the tunable capacitor for different angle $\theta_{0}{ }^{\prime}$

\begin{tabular}{ll}
\hline$\theta_{0}^{\prime}(\mathrm{deg})$ & TR $(\%)$ \\
\hline 5 & 29 \\
7 & 39 \\
10 & 52 \\
13 & 64 \\
\hline
\end{tabular}

\subsubsection{Influence of the pitch}

In this part, the influence of the interdigital angle is studied (pitch). Three cases have been investigated: fingers of 5,7 and $9^{\circ}$ with separating angle of 7,9 and $11^{\circ}$, respectively. Figure 4 shows the capacitance versus the angular displacement for different values of pitch.

Table 2 shows the tuning range for these three cases. We can see that the tuning range is the most interesting for structures with fingers of 9 spaced by $11^{\circ}$. With this design we can obtain a maximum tuning range of $60 \%$.

\subsubsection{Influence of the non fragmented area}

We keep the optimal angle $\theta_{0}{ }^{\prime}=13^{\circ}$. The pitch is $13^{\circ}$. We tried to minimize the area of the non fragmented

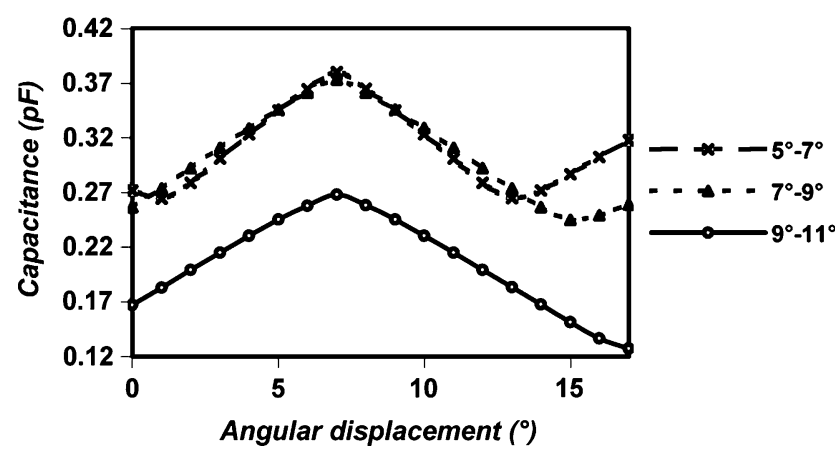

Fig. 4 Capacitance versus angular displacement for different value of pitch

Table 2 Tuning range of the tunable capacitor for different value of pitch

\begin{tabular}{lll}
\hline $\begin{array}{l}\theta_{0}{ }^{\prime} \\
(\mathrm{deg})\end{array}$ & $\begin{array}{l}\text { Pitch } \\
(\mathrm{deg})\end{array}$ & $\begin{array}{l}\text { TR } \\
(\%)\end{array}$ \\
\hline 5 & 7 & 40 \\
7 & 9 & 46 \\
9 & 11 & 60 \\
\hline
\end{tabular}

Table 3 Tuning range of the tunable capacitor for different value of $R_{2}$

\begin{tabular}{cl}
\hline$R_{2}(\mu \mathrm{m})$ & TR $(\%)$ \\
\hline 195 & 39.5 \\
200 & 28.3 \\
205 & 28 \\
\hline
\end{tabular}


part, by using a ring of $5 \mu \mathrm{m}$ width as the middle part instead of a full circle. Then, we simulated the capacitance versus angular displacement for different values of $R_{2}$. Table 3 shows the tuning range for three values of $R_{2}$.

To minimize the fringing capacitance and increase the tuning range, one solution is to minimize the non fragmented area. The drawback in this case is that the total capacitance is diminishing.

\subsubsection{Conclusion of the design optimization}

To obtain a maximum tuning range, we have to minimize the fringing capacitance. For that, three possible approaches exist:

- Increase the angle $\theta_{0}{ }^{\prime}$

- Increase the angle between two fingers

- Minimize the non fragmented area

Finally, we find a new optimized design with a tuning range of more than $100 \%(110 \%)$. Figure 5 shows the capacitance versus angular displacement for fingers of 9 separated by $11^{\circ}$ and $R_{2}=195 \mu \mathrm{m}$.

\section{Fabrication using polyimide sacrificial layer}

Figure 6 depicts the simplified successive steps of the process with AlSi $4 \%$. The silicon substrate is p-type with resistivity larger than $8 \mathrm{k} \Omega \mathrm{cm}$.

This process needs three levels of masks and one chemical-mechanical polishing step (CMP). First a silicon dioxide layer (with a thickness of $2 \mu \mathrm{m}$ ) is grown in a wet atmosphere as shown in Fig. 6a. The next step, as shown in Fig. 6b, is the deposition of AlSi $1 \%$ bottom electrode $(1 \mu \mathrm{m})$. The patterning of AlSi $1 \%$ metal layer is performed by a chlorine-based plasma chemistry (Fig. 6b). Then, a spin-on polyimide

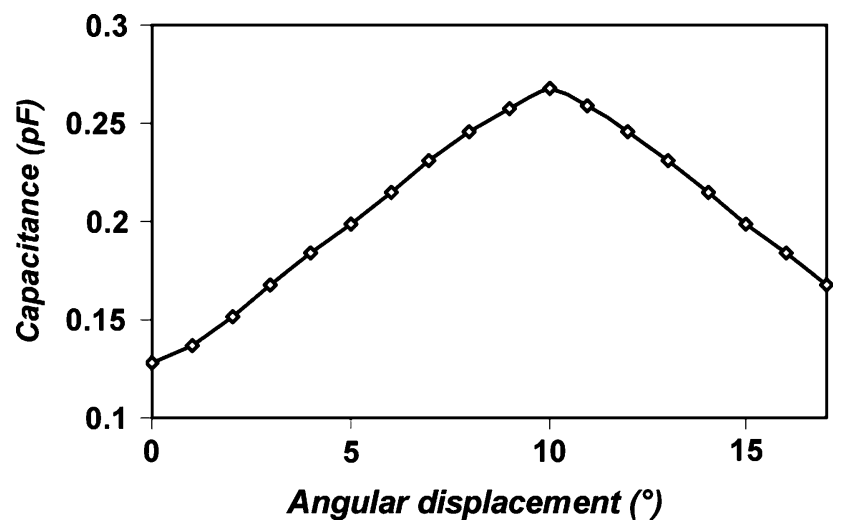

Fig. 5 Capacitance versus angular displacement (TR 110\%)

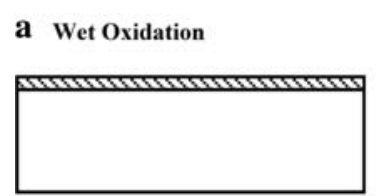

b AISi $1 \%$ Sputtering and Patterning (plasma $\mathrm{Cl}_{2} / \mathrm{BCl}_{3}$ )

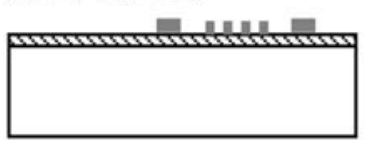

c Polyimide Deposition and CMP (Sacrificial Layer)

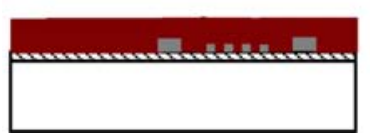

d Polyimide Patterning (Plasma $\mathrm{O}_{2}$ )

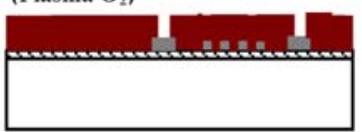

e AISi $4 \%$ Sputtering and Patterning (plasma $\mathrm{Cl}_{3} / \mathrm{BCl}_{3}$ )

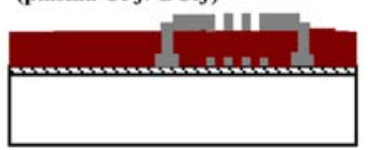

f Polyimide release (Plasma $\mathrm{O}_{2}$ )

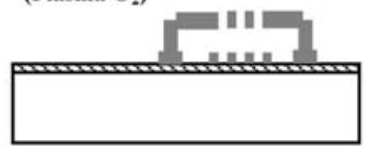

Fig. 6 Fabrication process of fragmented AlSi 4\% tunable capacitor using polyimide sacrificial layer

layer (pyralin PI-2610 from Dupont) defines the sacrificial layer (Fig. 6c). We use a solution of 3-aminopropyltriethoxysilane on isopropyl alcohol as adhesion promoter before the deposition and a long post-deposition bake of $200^{\circ} \mathrm{C} / 1 \mathrm{~h}$ followed by $300^{\circ} \mathrm{C} /$ $1 \mathrm{~h}$ in nitrogen atmosphere to have a solvent-free polyimide film (Pisani et al. 2003). A CMP is needed to level the sacrificial layer (Fig. 6c). Then, a step consists in etching through the polyimide layer (plasma $\mathrm{O}_{2}$ ) to prepare the mechanical anchors to the bottom electrode for the suspended electrode (Fig. 6d). The next step of the etching through the polyimide layer (plasma $\mathrm{O}_{2}$ ) to prepare the mechanical anchors to the bottom electrode for the suspended electrode (Fig. 6d). The next step of the process consists in metal layer deposition AlSi $4 \%(4 \mu \mathrm{m})$ by sputtering (Fig. 6e). The patterning of AlSi $4 \%$ metal layer is performed by a chlorine-based plasma chemistry (Fig. 6e). Then the suspended metal membranes are released by dry etching of the polyimide sacrificial layer in oxygen plasma $\left(\mathrm{O}_{2}\right)$ as shown in Fig. $6 \mathrm{f}$.

\section{Characterization}

Figure 7 shows an SEM image of the MEMS tunable capacitor with electrothermal actuator. Figure 8 shows the top fragmented electrode. Figure 9 shows a cross section of $1 \mu \mathrm{m}$ AlSi $1 \%+1.2 \mu \mathrm{m}$ air gap $+4 \mu \mathrm{m}$ AlSi $4 \%$.

For our fabricated MEMS capacitors (Figs. 7, 8, 9) the input power need for actuation ranges from 0 to $300 \mathrm{~mW}$, which turns into an operating voltage range of $0-1.2 \mathrm{~V}$. At zero voltage, the bottom and top elec- 


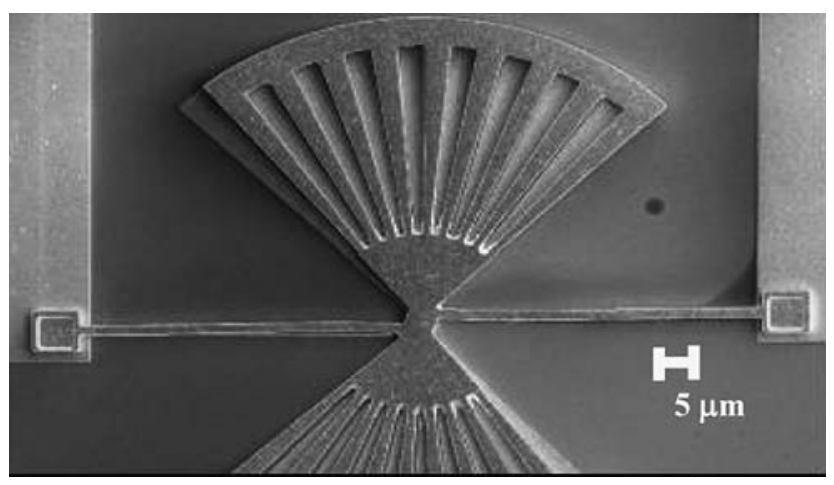

Fig. 7 SEM image of the MEMS tunable capacitor with electrothermal actuator

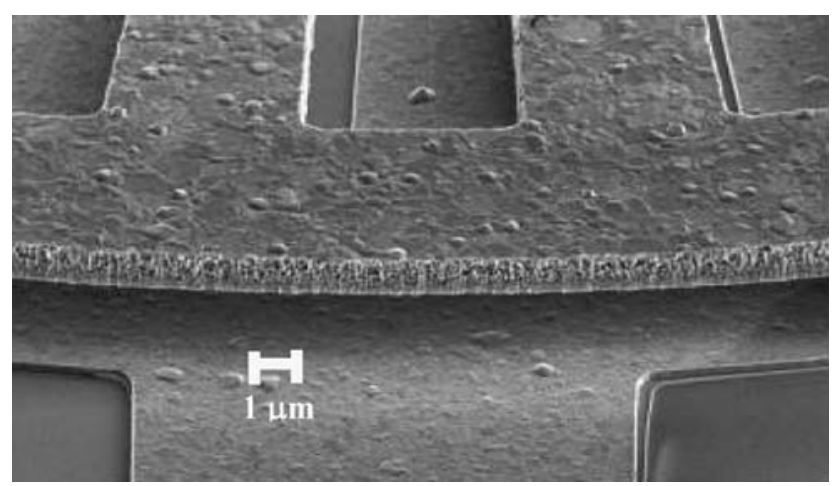

Fig. 8 SEM image of the top fragmented electrode

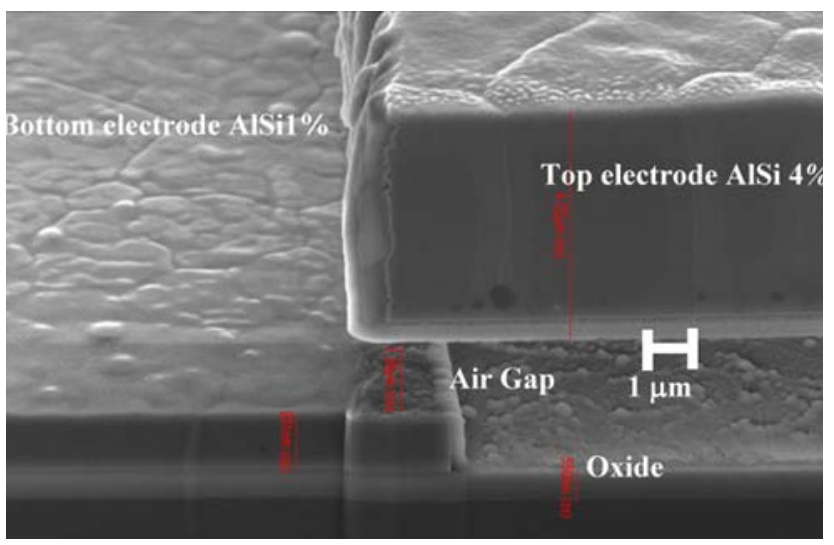

Fig. 9 Cross section of $1 \mu \mathrm{m}$ AlSi $1 \%+1.2 \mu \mathrm{m}$ air gap $+4 \mu \mathrm{m}$ AlSi 4\%

trode are intentionally misaligned. When increasing the applied voltage, the capacitance decreases and increases again (Fig. 10).

The measurements have been carried out by extracting the capacitance from S-parameter measurements made in the $2-5 \mathrm{GHz}$ range (see also Fig. 11) with a network analyzer HP 8719 D. We

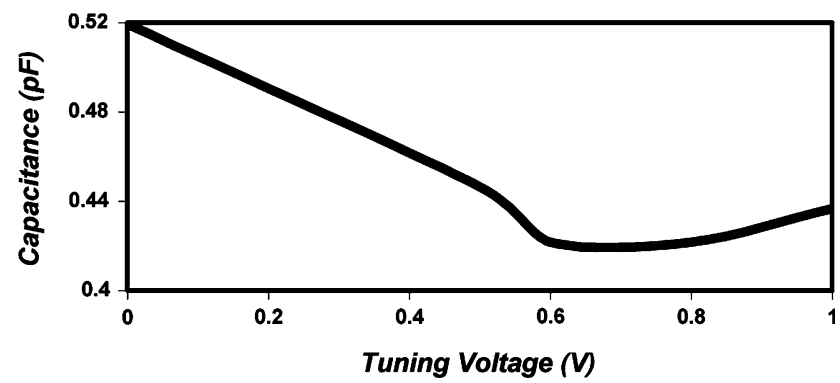

Fig. 10 Capacitance versus tuning voltage (experiment)

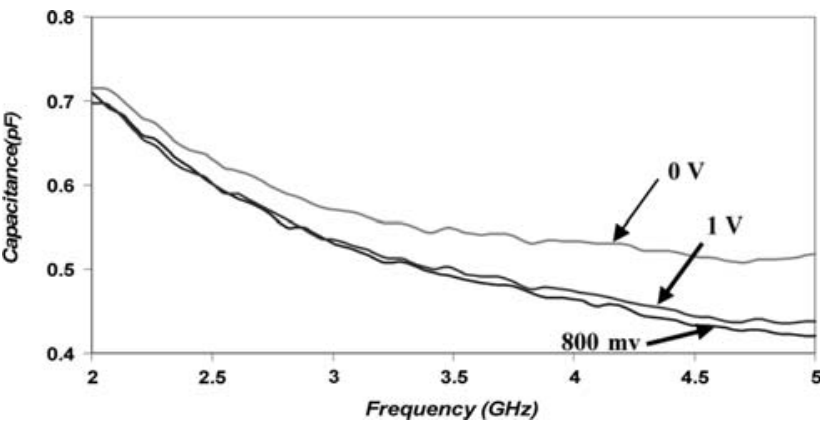

Fig. 11 Capacitance versus frequency (experiment)

obtain a maximum rotation near $1.2 \mathrm{~V}$ and $299 \mathrm{~mA}$ : the bottom and top electrode being aligned.

However, this maximum displacement is limited by buckling in the arms at high temperature $\left(>400^{\circ} \mathrm{C}\right.$, Fig. 12).

By combining electrical measurements with optical profilometry during actuation, the interpretation of Fig. 10 characteristic becomes clearer. Comparing the behavior of the real structure to the simulated device (Fig. 3), we can observe the following:

- The general trend shown by FEA, decrease of the capacitance and then increase, is reproduced in the experiment.

- The value of the measured capacitance is higher than in simulation or that predicted analytically. This could be attributed to the influence of the parasitic capacitances. Further, experiments should clarify this issue.

- At $1 \mathrm{~V}$ of polarization, the profilometer shows that the bottom and top electrodes are aligned. So, we would expect to get the highest capacitance as predicted by FEA and analytical model. However, experimental data show that although electrodes are aligned, the capacitance is lower than for $0 \mathrm{~V}$. In addition, profilometer measurements during actuation show that the structure begins to move out of plane even for a $0.6 \mathrm{~V}$ bias, increasing the gap and therefore decreasing the total capacitance (Fig. 10, Table 4). 

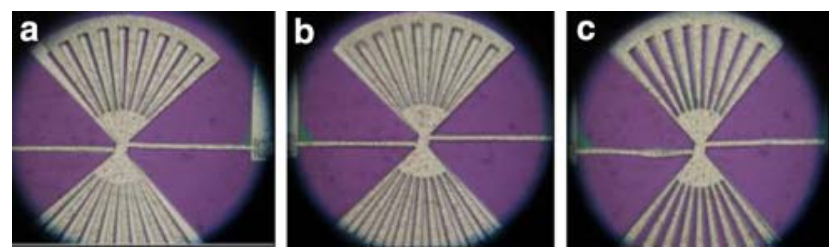

Fig. 12 Microscope picture of the structure. a under zero voltage, b under $0.8 \mathrm{~V}$ and $270 \mathrm{~mA}$, c under $1.2 \mathrm{~V}$ and $299 \mathrm{~mA}$

Table 4 Vertical displacement relative to initial position

\begin{tabular}{lll}
\hline $\begin{array}{l}\text { Applied } \\
\text { voltage } \\
(\mathrm{V})\end{array}$ & $\begin{array}{l}\text { Vertical displacement } \\
\text { relative to initial } \\
\text { position }(\mu \mathrm{m}) \\
\text { (middle part) }\end{array}$ & $\begin{array}{l}\text { Vertical displacement } \\
\text { relative to initial } \\
\text { position }(\mu \mathrm{m}) \\
\text { (extreme part) }\end{array}$ \\
\hline 0.6 & 7 & 0.1 \\
0.8 & 8.5 & 0.1 \\
0.9 & 11.8 & 0.8 \\
\hline
\end{tabular}

The reason for this behavior can be found in the mechanics of the structure. The central part of the top electrode is free to move vertically, so the total suspended length of the actuation beams becomes quite considerable. Thermal stress in the beams, which is compressive in our case, increases with voltage, and as a result when this stress goes beyond the critical value for buckling, the whole structure moves up instead of turning. A solution to this problem has been found by changing the position of the actuators and optimization of their design is under way. Figure $13 \mathrm{a}$ and $\mathrm{b}$ show the profile measurement of the top electrode, at 0 and $0.9 \mathrm{~V}$, respectively (Fig. 13).

\section{Conclusions}

In this paper, we validate for the first time the principle of the rotational electro-thermal actuator for MEMS tunable capacitors showing 30\% experimental tuning range. The experimental maximum angle is obtained at $7^{\circ}$, so less than one period. The experimental tuning range is somewhat smaller than the
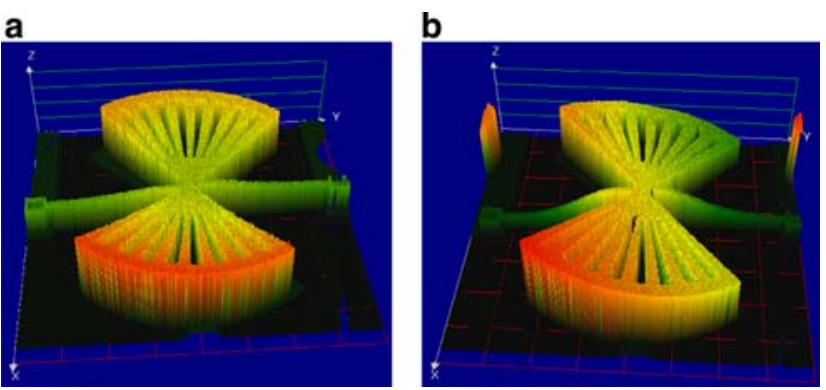

Fig. 13 Profile measurement of the top electrode. a under zero voltage, b under $0.9 \mathrm{~V}$. Vertical displacement occurs increasing the gap and decreasing the total capacitance

$70 \%$ predicted by simulation and the analytical model developed. We attribute this to the influence of parasitic capacitances.

Optimization of the design has been studied. By implementing certain design changes a tuning range of the capacitance of more than $100 \%$ should be obtainable. The MEMS architecture based on rotational actuation and fragmented electrodes does not suffer from pull in limiting effects, characteristic of conventional tunable capacitors, and offers a practical solution for future above-IC MEMS varicaps.

Acknowledgments Thanks to the EPFL-CMI staff for the support given on the technological steps development.

\section{References}

Dec A, Suyama K (1998) Micromachined electro-mechanically tunable capacitors and their applications to RF IC's. IEEE Trans Microw Theory Tech 46(12):2587-2596

Liu Q, Huang QA (2001) Micromachined variable capacitors with laterally positioned suspended plates. Proc SPIE 4601:20-24

Pisani MB, et al (2003) Copper/polyimide fabrication process for above-IC integration of high quality factors inductors. Microelectron Eng 73-74:474-479

Yoon JB, Nguyen TC (2000) A high Q tunable micromechanical capacitor with movable dielectric for RF Applications. IEDM 00-489

Young DJ, Boser BE (1996) A micromachined variable capacitor for monolithic low-noise VCOS. Solid-State Sensor and Actuator Workshop, pp 86-89 